\title{
REVIEW
}

\section{Clinical management for small bowel of Crohn's disease in the treat-to-target era: now is the time to optimize treatment based on the dominant lesion}

\author{
Kenji Watanabe \\ Center for Inflammatory Bowel Disease, Division of Internal Medicine, Hyogo College of Medicine, Nishinomiya, Japan
}

A treat-to-target strategy, in which treatment is continuously adjusted according to the results of scheduled objective monitoring, is optimal for patients with Crohn's disease (CD) in the era of biologics. The small bowel is a common site of intractable CD, which may result from multiple strictures or expanding lesions. To improve the prognosis of patients with small bowel CD, lesions should be proactively monitored within the subclinical phase. Objective assessment of small bowel lesions is technically difficult, however, due to the relatively poor correlation between endoscopic activity and clinical symptoms or biomarker titers. The presence of proximal small bowel lesions and asymptomatic "Real Silent CD" must be considered. Endoscopy remains the gold standard to assess these lesions. In clinical practice, the advantages and disadvantages of each imaging modality and biomarker must be carefully weighed for appropriate application and reliable monitoring. The prevalence of small bowel lesions depends on the precision of the imaging modality used for detection. Clinical management should be based on the dominant location of the intestinal lesions rather than classical classification. Optimal strategies for detecting and treating small bowel lesions in patients with CD must be developed utilizing reliable, precise, and objective monitoring. (Intest Res 2020;18:347-354)

Key Words: Crohn disease; Treat-to-target; Small bowel; Silent Crohn's disease; Dominant

\section{INTRODUCTION}

The number of patients with inflammatory bowel disease (IBD) in Asia is increasing annually. ${ }^{1,2}$ Asia will therefore be important toward advancing the global IBD field. Asian and Western IBD patients differ in both their genetic backgrounds and several clinical features. ${ }^{2,3}$ As such, clinical trials for newly developed drugs should confirm the efficacy and safety in both Asian and Western patients. ${ }^{4-6}$

Crohn's disease (CD) is a progressive disease, but patient

Received February 26, 2020. Revised May 20, 2020. Accepted June 8, 2020. Correspondence to Kenji Watanabe, Center for Inflammatory Bowel Disease, Division of Internal Medicine, Hyogo College of Medicine, 1-1 Mukogawa-cho, Nishinomiya 663-8501, Japan. Tel: +81-798-45-6663,

Fax:+81-798-45-6790, E-mail: ke-watanabe@hyo-med.ac.jp prognosis can be improved using a treat-to-target strategy with continuous scheduled objective monitoring. ${ }^{7.8}$ In contrast to ulcerative colitis, the gastrointestinal lesions of CD can occur anywhere from the mouth to the anus. Therefore, it is important to assess the existence, activity, and complications in a broad area compared over long-term periods in clinical management. The small bowel (SB) is an especially common location for CD lesions, making objective monitoring more difficult for CD lesions than for colonic lesions.

Tight control of SB lesions is a key factor in the clinical management of $\mathrm{CD}$. Therefore, the strategies and techniques for diagnosing, monitoring, and treating SB lesions in patients with CD must be optimized. ${ }^{9}$ 


\section{IMPORTANCE OF SB LESIONS IN PATIENTS WITH CD FOR CLINICAL PRACTICE}

Why are SB lesions in patients with CD important in clinical practice? de Barros et al. ${ }^{10}$ demonstrated the importance of the location of SB lesions according to the Montreal classification with a 65.2-month follow-up. An ileal (L1) lesion was more significantly associated with a complicated disease course (relative risk [RR], 1.64; 95\% confidence interval [CI], 1.10-1.75) and stricturing behavior (RR, 2.11; 95\% CI, 1.20-3.69) than a colonic (L2) lesion. Further, L1 lesions are also associated with poorer abdominal surgery outcomes compared to ileocolonic (L3) lesions (RR, 1.68; 95\% CI, 1.13-2.50). In another investigation using data obtained from the REACT trial involving 1,898 CD patients, SB lesions were more strongly associated with CD-related surgery than colonic lesions (odds ratio [OR], 2.03; 95\% CI, 1.16-3.57). ${ }^{11}$ At our institute, SB lesions are the lesions most frequently responsible for surgery in patients with $\mathrm{CD}^{12}$ It is critical to recognize the importance of SB lesions in the clinical management of patients with $\mathrm{CD}$.

\section{FEATURES OF SB LESIONS IN PATIENTS WITH} CD

"Silent CD" is a term applied to asymptomatic CD patients with active SB lesions. Active SB lesions may be present even during asymptomatic clinical remission or when the patient has low levels of inflammatory markers. In 2015, Click et al. ${ }^{13}$ linked elevated C-reactive protein (CRP) levels to "Silent CD" in asymptomatic CD patients. They evaluated 351 asymptomatic CD patients with a 2-year observation period by classifying them into an elevated CRP group and a normal CRP group. CRP elevation was significantly associated with an increased risk of hospitalization (adjusted hazard ratio, 2.12; 95\% CI, 1.13-3.98) in multivariate survival analysis. The same group also demonstrated by using the Lémann Index that elevated CRP is independently associated with 7 -fold greater worsening of the disease trajectory (OR, 6.93; 95\% CI, 2.44-19.67) compared with a stable disease trajectory ${ }^{14,15}$ In my opinion, however, asymptomatic "Silent CD" with CRP elevation is not actually "Silent CD" because the increase in CRP, which is commonly measured in daily clinical practice, alerts the physician (especially IBD specialists) to the presence of an active lesion. We should be aware of "Real Silent CD" in which CRP levels are not elevated. Approximately $50 \%$ of patients with normal CRP levels have an active lesion (as assessed by endoscopy) and
$30 \%$ of patients are hospitalized for intestinal resection. ${ }^{13}$

Korean investigators reported that half of the disease location sites in CD patients with normal CRP levels were in the ileum. ${ }^{16}$ All operations related to ileocolonic or colonic lesions could be predicted by physicians whereas only $75 \%$ of the operations related to ileal lesions could be predicted. These authors concluded that CRP is less useful as a disease activity marker in patients with ileal CD than in those with ileocolonic or colonic CD. A Spanish group studied fecal calprotectin (FCP) levels in CD patients who had undergone ileocolonic resection. ${ }^{17}$ In that study, the group with a Rutgeerts score of i2 had a median FCP level of 166.5 (interquartile range [IQR], 64.8316.3) and a median CRP level of 0.45 (IQR, 0.11-0.62), and the group with an i3 score had a median FCP of 98.5 (IQR, 63625) and a median CRP of 0.1 (IQR, 0.05-0.73).

Thus, CD patients with active SB lesions can exhibit "Real Silent CD" coupled with a normal CRP or FCP range. ${ }^{18}$ For this reason, I emphasize the importance of assessing SB lesions in patients with CD using imaging modalities such as endoscopy, magnetic resonance enterography (MRE), or ultrasound. The International Organization for the Study of IBD (IOIBD) states that although CRP and FCP are useful adjunctive biomarkers, they should not be used as treatment targets because normalization of these biomarkers alone is not sufficient. ${ }^{19}$

\section{OBJECTIVE MONITORING OF SB LESIONS IN PATIENTS WITH CD}

Continuous adjustment of treatment depending on the results of scheduled objective monitoring is the key concept of the "treat-to-target" strategy. ${ }^{8}$ The IOIBD states that "Endoscopy remains the gold standard for assessing location, depth, and extent of inflammatory mucosal lesions in CD. ${ }^{19}$ When endoscopy cannot adequately evaluate inflammation, however, cross-sectional imaging is necessary. In other words, crosssectional imaging and evaluation of biomarkers such as CRP and FCP are useful follow-up monitoring methods. Measurement of CRP or FCP levels cannot indicate the deformity, localization, distribution, or disability of CD lesions, but can provide complementary information. The appropriate method should be used at the appropriate time by considering the strengths and limitations of each monitoring method.

Monitoring CD by only conventional ileocolonoscopy is insufficient in cases of proximal ileum dominant or negative findings of conventional ileocolonoscopy at the initial diagnosis. ${ }^{20,21}$ Fig. 1 shows the diagnostic strategy for achieving a definite di- 


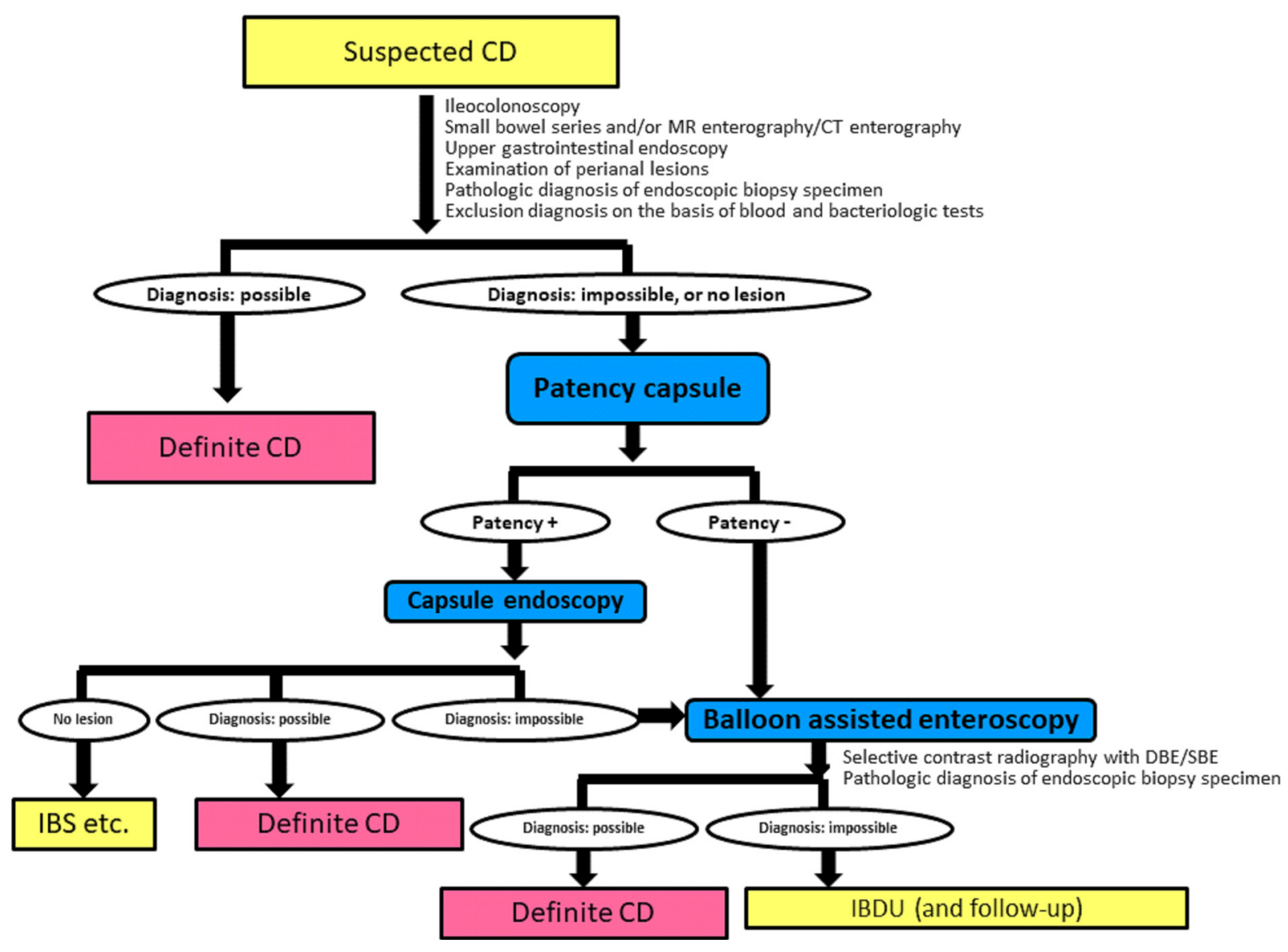

Fig. 1. Flowchart of the diagnostic strategy for achieving a definite diagnosis in difficult cases of patients with suspected Crohn's disease (CD). MR, magnetic resonance; $C T$, computed tomography; IBS, irritable bowel syndrome; DBE, double balloon enteroscopy; SBE, single balloon enteroscopy; IBDU, inflammatory bowel disease unclassified. Modified from Watanabe K, et al. Nihon Shokakibyo Gakkai Zasshi 2015;112:1259-1269, with permission from the Japanese Society of Gastroenterology. ${ }^{12}$

agnosis in difficult cases of patients with suspected CD using a patency capsule and subsequent capsule endoscopy (CE) or balloon-assisted enteroscopy (BAE) such as double balloon enteroscopy or single balloon enteroscopy. ${ }^{12}$ This algorithm is applicable to patients with suspected CD. Ultrasound is a noninvasive monitoring modality for $\mathrm{CD}$, but visualization of the proximal ileum is relatively difficult for technical reasons and the quality of visualization depends on the skill of the ultrasonographer. ${ }^{22,23}$ MRE has several advantages and is globally recognized as a useful monitoring modality for the SB in patients with $\mathrm{CD}^{24,25} \mathrm{MRE}$ is a noninvasive and radiation-free method for visualizing the layers of the intestinal wall and also permits visualization of extraintestinal findings in the abdomen. MRE, however, also has disadvantages. González-Suárez et al. ${ }^{26}$ reported that CE is superior to MRE for detecting lesions in all parts of the SB (jejunum, ileum, and terminal ileum). Further, MRE is less sensitive for detecting stenosis compared with BAE, especially for a thin stenosis without wall thickening, e.g., stricture formation as the result of mucosal healing after the administration of an anti-tumor necrosis factor (TNF)- $\alpha$ agent. ${ }^{27}$ Cross-sectional imaging, such as ultrasound, computed tomography, enterography, or MRE, is superior to CE or BAE for assessing transmural inflammation. Transmural healing is a more ideal treatment goal beyond complete endoscopic mucosal healing in CD clinical practice. While complete endoscopic mucosal healing in the whole gastrointestinal tract is still the ideal goal, it is not easy to achieve in most CD patients, even in the era of biologics.

SB endoscopy is the most sensitive imaging modality for visualizing SB lesions in patients with $\mathrm{CD}^{28}$ Proactive management of CD patients was recently recognized as the optimal approach for avoiding disability and changing the natural progression of the disease compared with reactive management. ${ }^{29}$ From this perspective, endoscopic monitoring is an ideal ap- 
proach for proactively detecting changes in the disease progress within the subclinical phase. In 1990, Rutgeerts et al. ${ }^{30}$ demonstrated that endoscopic recurrence develops prior to clinical recurrence in the neoterminal ileum after ileocecal resection. Endoscopic assessment and treating to target increase the likelihood of mucosal healing in patients with $\mathrm{CD} .{ }^{31}$ Of course, endoscopic examination of the SB has some limitations, such as potential capsule retention in CE, inability to achieve deep insertion in BAE due to severe adhesion or stenosis, and risk of perforation. ${ }^{9,32-35}$ Appropriate use and secure confirmation of the functional patency of the intestinal tract using a patency capsule is essential to avoid capsule retention in CE. ${ }^{9,34}$ In cases with stricture, ultrathin colonoscopy is sometimes useful for passing the stricture and observing the proximal lumen beyond the stricture. ${ }^{36}$ Deep insertion should be cautiously performed in cases with higher active ileal lesions, especially those with longitudinal ulcers at the mesenteric site. ${ }^{9.32}$

Some investigators or physicians mention invasiveness as a disadvantage of conventional ileocolonoscopy or BAE. While it is true that sedation is required for these endoscopic examinations, the balance between invasiveness and accuracy must be carefully considered. Inaccurate monitoring will provide insufficient information for decision-making in clinical practice. Each imaging modality and biomarker has its advantages and disadvantages. Objective monitoring procedures should be used appropriately on a case-by-case basis. Additionally, most patients will accept the risk of an invasive procedure if they are able to understand the value of the procedure for their clinical management. ${ }^{37}$ Comparisons of the endoscopic picture between before and after treatment will help to demonstrate the efficacy of the treatment to the patient.

\section{EFFICACY OF MEDICATION FOR SB LESIONS IN PATIENTS WITH CD}

Is the efficacy of pharmacologic treatment for ileal lesions in patients with CD inferior to that for colonic lesions? $?^{38-40}$ Reliable, precise, and objective procedures are needed to confirm the efficacy of each pharmacologic treatment for SB and colonic lesions. Further, for reliable translation from basic research, the mode of action for each drug must be carefully considered. ${ }^{41}$

Most previous studies analyzed the data of the lesion location according to the Montreal classification. ${ }^{42,43}$ Classification of the location, however, requires precise monitoring. More precise monitoring will likely result in an increase in the prevalence of SB lesion in patients with $\mathrm{CD}^{44-47}$ Therefore, I propose that clinical management and investigation depend on the dominancy of the location of CD lesions in the gastrointestinal tract. Recently, Dulai et al. ${ }^{48}$ suggested a new classification for CD as either ileum dominant (isolated ileal and ileocolonic) or isolated colonic disease. However, I would like to advance the concept of a novel classification that depends only on location dominancy, as follows: jejunal dominant (rare); proximal ileum dominant; terminal ileum dominant; expanded SB dominant, in which lesions are distributed in more than 2 sections in the SB; colon dominant; and expanded SB-colon dominant (Table 1). The precision of imaging modalities is rapidly improving. Therefore, proactive clinical management should be performed. My proposed classification according to the dominance of the location of CD lesions capitalizes on the more detailed information provided by modern imaging modalities. In fact, my preliminary data revealed that $50 \%$ of lesions responsible for surgery were located in the small bowel (proxi-

Table 1. Montreal Classification and Novel Classification According to The Dominant Lesion Location in the Gastrointestinal Tract in Patients with Crohn's Disease

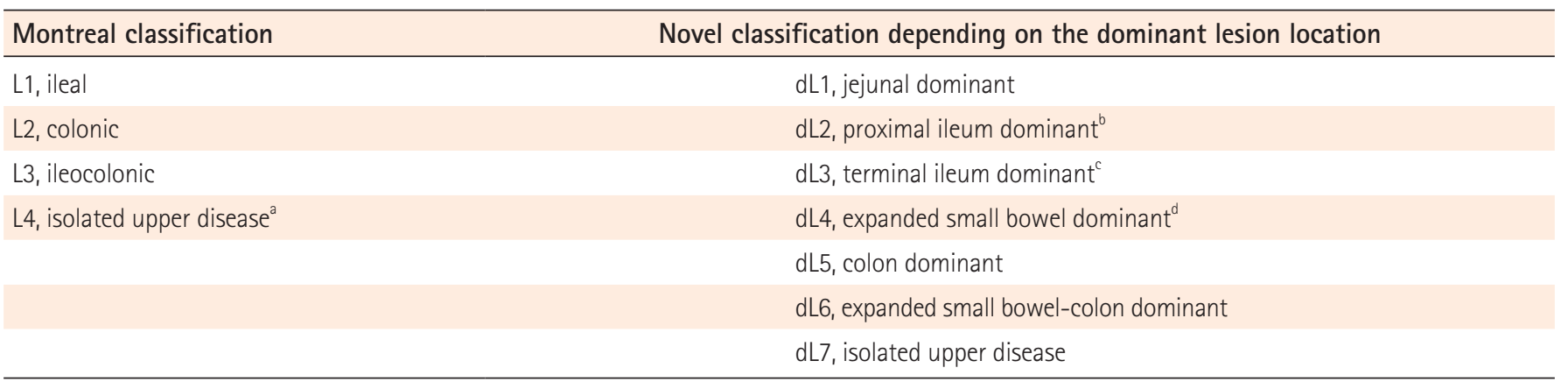

a 4 or $\mathrm{dL7}$ is a modifier that can be added to L1-L3 or dL1-dL6 when concomitant upper gastrointestinal disease is present.

bProximal ileum is defined as $>10 \mathrm{~cm}$ proximal to terminal ileum.

'Terminal ileum is defined as $\leq 10 \mathrm{~cm}$ from ileocecal valve.

dExpanded small bowel dominant is defined as lesions distributed in more than 2 sections in the small bowel. 
mal to the terminal ileum) compared with $25 \%$ located in the ileocecal region, including the terminal ileum. Further studies are needed to confirm the utility of this novel classification.

\section{ENDOSCOPIC BALLOON DILATATION AND SURVEILLANCE FOR SB LESIONS IN PATIENTS WITH CD}

BAE has some advantages for the treatment of SB strictures in clinical practice. Endoscopic balloon dilatation (EBD) is widely performed to avoid surgery in daily clinical practice. Strictures in the proximal ileum or jejunum can usually be dilated only by BAE. Hirai et al. ${ }^{49}$ investigated the efficacy and safety of EBD for SB strictures by BAE in a prospective multicenter study of patients with CD. Approximately $70 \%$ of the participants demonstrated short-term improvement in their symptoms and a larger dilatation diameter of the balloon was associated with the success of the procedure.

The short-term success of EBD depends on the patient selection with inclusion and exclusion criteria. ${ }^{9} \mathrm{~A}$ long stricture $(\geq 5 \mathrm{~cm})$, the presence of a fistula around the stricture, a severe angulated stricture, and a severe active lesion at the stricture site are contraindications for the procedure. Erosion at the stricture is not usually a contraindication, but the acceptable level of an active ulcer is controversial, as a shallow ulcer might not rule out dilation. Endoscopists should pay special attention to the presence of fissuring ulcers around the stricture site just before EBD by X-ray monitoring using a contrast agent. Fissuring ulcers are at very high-risk for perforation by EBD. The inclusion criteria for EBD are also controversial. EBD is indicated for CD patients with obstructive symptoms or a dilated intestine at the oral side of a stricture. CD patients with SB strictures without dilation at the oral side and no obstructive symptoms may undergo EBD at the discretion of the physician. In my clinical practice, strictures that cannot be passed through with an endoscope, even though without dilation at the oral side and without obstructive symptoms, are usually dilated to avoid the development of more severe strictures. Finally, multiple ileal strictures are often observed, and the number of strictures should be considered in the decision to perform EBD. To avoid surgery, all SB strictures should be dilated, but successful dilation of all SB strictures requires a highly skilled endoscopist due to the likelihood of severe adhesions.

The long-term efficacy of EBD is equally important for the patient's prognosis. Hirai et al. ${ }^{50}$ previously demonstrated a cumulative surgery-free rate of $79 \%$ at 2 years and $73 \%$ at 3 years after the initial EBD. The cumulative redilation-free rate after initial EBD was $64 \%$ at 2 years and $47 \%$ at 3 years. The success of endoscopic dilation for all SB strictures and control of inflammation by medical treatment are key factors for avoiding hospitalization and surgery in the long-term. Ultimately, a treatto-target strategy with continuous precise objective monitoring and subsequent adjustment of the treatment content according to the results of the monitoring is important to avoid disabling states such as stricture formation. ${ }^{14}$ In contrast, after stricture formation, BAE or surgery should be selected on a case-by-case basis because multiple factors, including the clinical characteristics, clinical treatment history, imaging findings of the stricture, and skills of the endoscopist, must be considered in the decision-making process. ${ }^{51}$

CD-related SB cancer has a relatively low incidence in East Asia compared with Western countries. ${ }^{52}$ BAE can be used to obtain biopsy tissue for surveillance. The endoscopic features of SB cancer are not yet well established because poorly differentiated adenocarcinoma or mucinous carcinoma is often observed in CD patients with CD-related SB cancer. The endoscopic findings of these cancers may differ from those of well-differentiated adenocarcinoma, similar to what is observed in colonic lesions in patients with ulcerative colitis. ${ }^{53} \mathrm{SB}$ strictures that are intractable to EBD are a risk factor and biopsy tissue should be obtained for surveillance. ${ }^{54}$

\section{CONCLUSIONS}

Continuous and scheduled precise objective monitoring and adjustment of treatments are key factors in the treat-to-target strategy. Even if the short-term outcome is not significantly different from that of a non-treat-to-target group, the long-term outcome by the treat-to-target strategy should improve compared with the non-treat-to-target strategy. ${ }^{55}$ For example, an adequate serum concentration of anti-TNF- $\alpha$ agent is variable depending on the situation: for remission induction, remission maintenance, or disease activity. ${ }^{56}$ An optimal treatment strategy for SB lesions in patients with CD must be developed on the basis of reliable data obtained by precise and objective monitoring. Clinical management should be based on the dominant location of the intestinal lesions.

\section{FINANCIAL SUPPORT}

The author received no financial support for the research, authorship, and/or publication of this article. 


\section{CONFLICT OF INTEREST}

No potential conflict of interest relevant to this article was reported.

\section{AUTHOR CONTRIBUTION}

Writing and approval of final manuscript: Watanabe $\mathrm{K}$.

\section{ORCID}

Watanabe K

$$
\text { https://orcid.org/0000-0002-3781-4724 }
$$

\section{REFERENCES}

1. Murakami Y, Nishiwaki Y, Oba MS, et al. Estimated prevalence of ulcerative colitis and Crohn's disease in Japan in 2014: an analysis of a nationwide survey. J Gastroenterol 2019;54:10701077.

2. Ng WK, Wong SH, Ng SC. Changing epidemiological trends of inflammatory bowel disease in Asia. Intest Res 2016;14:111119 .

3. Liu JZ, van Sommeren S, Huang H, et al. Association analyses identify 38 susceptibility loci for inflammatory bowel disease and highlight shared genetic risk across populations. Nat Genet 2015;47:979-986.

4. Watanabe K, Motoya S, Ogata H, et al. Effects of vedolizumab in Japanese patients with Crohn's disease: a prospective, multicenter, randomized, placebo-controlled phase 3 trial with exploratory analyses. J Gastroenterol 2020;55:291-306.

5. Miyazaki T, Watanabe K, Kojima K, et al. Efficacies and related issues of ustekinumab in Japanese patients with Crohn's disease: a preliminary study. Digestion 2020;101:53-59.

6. Morita Y, Imai T, Bamba S, et al. Clinical relevance of innovative immunoassays for serum ustekinumab and anti-ustekinumab antibody levels in Crohn's disease. J Gastroenterol Hepatol 2020;35:1163-1170.

7. Peyrin-Biroulet L, Sandborn W, Sands BE, et al. Selecting Therapeutic Targets in Inflammatory Bowel Disease (STRIDE): determining therapeutic goals for treat-to-target. Am J Gastroenterol 2015;110:1324-1338.

8. Colombel JF, Panaccione R, Bossuyt P, et al. Effect of tight control management on Crohn's disease (CALM): a multicentre, randomised, controlled phase 3 trial. Lancet 2018;390:27792789.

9. Yamamoto H, Ogata H, Matsumoto T, et al. Clinical practice guideline for enteroscopy. Dig Endosc 2017;29:519-546.

10. de Barros KSC, Flores C, Harlacher L, Francesconi CFM. Evolution of clinical behavior in Crohn's disease: factors associated with complicated disease and surgery. Dig Dis Sci 2017;62: 2481-2488.

11. Guizzetti L, Zou G, Khanna R, et al. Development of clinical prediction models for surgery and complications in Crohn's disease. J Crohns Colitis 2018;12:167-177.

12. Watanabe K, Hosomi S, Noguchi A, et al. Significances and issues for capsule endoscopy in patients with Crohn's diseasetoward the appropriate use. Nihon Shokakibyo Gakkai Zasshi 2015;112:1259-1269.

13. Click B, Vargas EJ, Anderson AM, et al. Silent Crohn's disease: asymptomatic patients with elevated C-reactive protein are at risk for subsequent hospitalization. Inflamm Bowel Dis 2015; 21:2254-2261.

14. Pariente B, Cosnes J, Danese S, et al. Development of the Crohn's disease digestive damage score, the Lémann score. Inflamm Bowel Dis 201 1;17:1415-1422.

15. Bhattacharya A, Rao BB, Koutroubakis IE, et al. Silent Crohn's disease predicts increased bowel damage during multiyear follow-up: the consequences of under-reporting active inflammation. Inflamm Bowel Dis 2016;22:2665-2671.

16. Yang DH, Yang SK, Park SH, et al. Usefulness of C-reactive protein as a disease activity marker in Crohn's disease according to the location of disease. Gut Liver 2015;9:80-86.

17. Verdejo C, Hervías D, Roncero O, et al. Fecal calprotectin is not superior to serum C-reactive protein or the Harvey-Bradshaw index in predicting postoperative endoscopic recurrence in Crohn's disease. Eur J Gastroenterol Hepatol 2018;30:15211527.

18. Nakamura S, Imaeda H, Nishikawa H, et al. Usefulness of fecal calprotectin by monoclonal antibody testing in adult Japanese with inflammatory bowel diseases: a prospective multicenter study. Intest Res 2018;16:554-562.

19. Peyrin-Biroulet L, Panés J, Sandborn WJ, et al. Defining disease severity in inflammatory bowel diseases: current and future directions. Clin Gastroenterol Hepatol 2016;14:348-354.

20. Dionisio PM, Gurudu SR, Leighton JA, et al. Capsule endoscopy has a significantly higher diagnostic yield in patients with suspected and established small-bowel Crohn's disease: a meta-analysis. Am J Gastroenterol 2010;105:1240-1248.

21. Enns RA, Hookey L, Armstrong D, et al. Clinical practice guidelines for the use of video capsule endoscopy. Gastroenterology 2017;152:497-514.

22. Kucharzik T, Wittig BM, Helwig U, et al. Use of intestinal ultra- 
sound to monitor Crohn's disease activity. Clin Gastroenterol Hepatol 2017;15:535-542.

23. Aloi M, Di Nardo G, Romano G, et al. Magnetic resonance enterography, small-intestine contrast US, and capsule endoscopy to evaluate the small bowel in pediatric Crohn's disease: a prospective, blinded, comparison study. Gastrointest Endosc 2015;81:420-427.

24. Lamb CA, Kennedy NA, Raine T, et al. British Society of Gastroenterology consensus guidelines on the management of inflammatory bowel disease in adults. Gut 2019;68(Suppl 3): s1-s106.

25. Maaser C, Sturm A, Vavricka SR, et al. ECCO-ESGAR Guideline for Diagnostic Assessment in IBD Part 1: initial diagnosis, monitoring of known IBD, detection of complications. J Crohns Colitis 2019;13:144-164.

26. González-Suárez B, Rodriguez S, Ricart E, et al. Comparison of capsule endoscopy and magnetic resonance enterography for the assessment of small bowel lesions in Crohn's disease. Inflamm Bowel Dis 2018;24:775-780.

27. Takenaka K, Ohtsuka K, Kitazume Y, et al. Comparison of magnetic resonance and balloon enteroscopic examination of the small intestine in patients with Crohn's disease. Gastroenterology 2014;147:334-342.

28. Solem CA, Loftus EV Jr, Fletcher JG, et al. Small-bowel imaging in Crohn's disease: a prospective, blinded, 4-way comparison trial. Gastrointest Endosc 2008;68:255-266.

29. Torres J, Burisch J, Riddle M, Dubinsky M, Colombel JF. Preclinical disease and preventive strategies in IBD: perspectives, challenges and opportunities. Gut 2016;65:1061-1069.

30. Rutgeerts P, Geboes K, Vantrappen G, Beyls J, Kerremans R, Hiele M. Predictability of the postoperative course of Crohn's disease. Gastroenterology 1990;99:956-963.

31. Bouguen G, Levesque BG, Pola S, Evans E, Sandborn WJ. Endoscopic assessment and treating to target increase the likelihood of mucosal healing in patients with Crohn's disease. Clin Gastroenterol Hepatol 2014;12:978-985.

32. Oshitani N, Yukawa T, Yamagami H, et al. Evaluation of deep small bowel involvement by double-balloon enteroscopy in Crohn's disease. Am J Gastroenterol 2006;101:1484-1489.

33. Esaki M, Matsumoto T, Watanabe K, et al. Use of capsule endoscopy in patients with Crohn's disease in Japan: a multicenter survey. J Gastroenterol Hepatol 2014;29:96-101.

34. Watanabe K, Ohmiya N, Nakamura M, Fujiwara Y. A prospective study evaluating the clinical utility of the tag-less patency capsule with extended time for confirming functional patency. Digestion. [published online ahead of print September 19,
2019]. https://doi.org/10.1159/000503027.

35. Tokuhara D, Watanabe K, Okano Y, et al. Wireless capsule endoscopy in pediatric patients: the first series from Japan. J Gastroenterol 2010;45:683-691.

36. Morimoto K, Watanabe K, Noguchi A, et al. Clinical impact of ultrathin colonoscopy for Crohn's disease patients with strictures. J Gastroenterol Hepatol 2015;30 Suppl 1:66-70.

37. Bewtra M, Fairchild AO, Gilroy E, et al. Inflammatory bowel disease patients' willingness to accept medication risk to avoid future disease relapse. Am J Gastroenterol 2015;1 10:1675-1681.

38. Beppu T, Ono Y, Matsui T, et al. Mucosal healing of ileal lesions is associated with long-term clinical remission after infliximab maintenance treatment in patients with Crohn's disease. Dig Endosc 2015;27:73-81.

39. Takenaka K, Fujii T, Suzuki K, et al. Small bowel healing detected by endoscopy in patients with crohn's disease after treatment with antibodies against tumor necrosis factor. Clin Gastroenterol Hepatol 2020;18:1545-1552.

40. Danese S, Sandborn WJ, Colombel JF, et al. Endoscopic, radiologic, and histologic healing with vedolizumab in patients with active Crohn's disease. Gastroenterology 2019;157:10071018.

41. Habtezion A, Nguyen LP, Hadeiba H, Butcher EC. Leukocyte trafficking to the small intestine and colon. Gastroenterology 2016;150:340-354.

42. Silverberg MS, Satsangi J, Ahmad T, et al. Toward an integrated clinical, molecular and serological classification of inflammatory bowel disease: report of a Working Party of the 2005 Montreal World Congress of Gastroenterology. Can J Gastroenterol 2005;19 Suppl A:5A-36A.

43. Satsangi J, Silverberg MS, Vermeire S, Colombel JF. The Montreal classification of inflammatory bowel disease: controversies, consensus, and implications. Gut 2006;55:749-753.

44. Hall B, Holleran G, McNamara D. Small bowel Crohn's disease: an emerging disease phenotype? Dig Dis 2015;33:42-51.

45. Cotter J, Dias de Castro F, Moreira MJ, Rosa B. Tailoring Crohn's disease treatment: the impact of small bowel capsule endoscopy. J Crohns Colitis 2014;8:1610-1615.

46. Papay P, Ignjatovic A, Karmiris K, et al. Optimising monitoring in the management of Crohn's disease: a physician's perspective. J Crohns Colitis 2013;7:653-669.

47. Flamant M, Trang C, Maillard O, et al. The prevalence and outcome of jejunal lesions visualized by small bowel capsule endoscopy in Crohn's disease. Inflamm Bowel Dis 2013;19:13901396.

48. Dulai PS, Singh S, Vande Casteele N, et al. Should we divide 
Crohn's disease into ileum-dominant and isolated colonic diseases? Clin Gastroenterol Hepatol 2019;17:2634-2643.

49. Hirai F, Andoh A, Ueno F, et al. Efficacy of endoscopic balloon dilation for small bowel strictures in patients with Crohn's disease: a nationwide, multi-centre, open-label, prospective cohort study. J Crohns Colitis 2018;12:394-401.

50. Hirai F, Beppu T, Takatsu N, et al. Long-term outcome of endoscopic balloon dilation for small bowel strictures in patients with Crohn's disease. Dig Endosc 2014;26:545-551.

51. Lan N, Stocchi L, Ashburn JH, et al. Outcomes of endoscopic balloon dilation vs surgical resection for primary ileocolic strictures in patients with Crohn's disease. Clin Gastroenterol Hepatol 2018;16:1260-1267.

52. Higashi D, Katsuno H, Kimura H, et al. Current state of and problems related to cancer of the intestinal tract associated with Crohn's disease in Japan. Anticancer Res 2016;36:37613766.
53. Matsumoto T, Iwao Y, Igarashi M, et al. Endoscopic and chromoendoscopic atlas featuring dysplastic lesions in surveillance colonoscopy for patients with long-standing ulcerative colitis. Inflamm Bowel Dis 2008;14:259-264.

54. Sogawa M, Watanabe K, Egashira Y, et al. Precise endoscopic and pathologic features in a Crohn's disease case with two fistula-associated small bowel adenocarcinomas complicated by an anal canal adenocarcinoma. Intern Med 2013;52:445449.

55. Vande Casteele N, Ferrante M, Van Assche G, et al. Trough concentrations of infliximab guide dosing for patients with inflammatory bowel disease. Gastroenterology 2015;148:13201329.

56. Watanabe K, Matsumoto T, Hisamatsu T, et al. Clinical and pharmacokinetic factors associated with adalimumab-induced mucosal healing in patients with Crohn's disease. Clin Gastroenterol Hepatol 2018;16:542-549. 